\title{
On the importance of thermo-elastic stressing in injection-induced earthquakes
}

\author{
Candela, T., \\ van der Veer, E.F., \\ Fokker, P.A. \\ TNO Applied Geosciences, P.O. Box 80015, 3508 TA Utrecht, Netherlands
}

\begin{abstract}
The sustainability of geothermal fields is based on a paradox. On one side, fractures are targeted on heat-flow improvement, and on the other side the same fractures are avoided because of induced-seismicity risk. In this context, we developed analytical approaches for estimating (1) thermo-poro-elastic stresses in a fractured geothermal system, and (2) seismicity rates based on the model of Dieterich (1994). We modeled cold water injected at a constant rate into a single fracture surrounded by hot impermeable layers. The rationale for focusing on one single isolated fracture was that flow in the vicinity of injection wells is often concentrated in a couple of fractures instead of being homogeneously distributed. Heat flow appeared to be dominated by advection inside the fracture and conduction outside it. Poro- and thermo-elastic stresses around the single fracture were estimated separately following two independent analytical approaches; and for any potential fault around the single fracture the induced Coulomb stress rates were resolved. The role of thermal stresses appeared to be the leading one. We show that thermal-stressing rates can induce an increase in the rate of seismicity of more than a thousand-fold at distances up to $200 \mathrm{~m}$ from the single fracture. Our fast forward models are suitable for data assimilation and they open the route for heat-flow optimization while keeping seismicity at a relatively low magnitude.
\end{abstract}

\section{Introduction}

The understanding of geothermal fields requires coupling between heat flow through fractures and induced seismicity: slip along fractures can enhance the heat flow but can also lead to large seismic events. The grail is to take advantage of fracture conductivity while keeping seismicity at a relatively low magnitude, thus avoiding risks of large earthquakes (Zang et al., 2013). Our present contribution targets a better understanding of this complex coupling between heat transfer, flow, fractures, and earthquakes. 
Flow through a fractured geothermal system is often modelled with complex numerical approaches, taking into account the interaction between multiple fractures (Taron et al., 2009; Izadi and Elsworth, 2010; McClure and Horne, 2010; Jalali, 2013). These models give detailed pictures of the Thermo-Hydro-Mechanical couplings, but the time-consuming nature of such models preclude processes and physical parameters validation and updating through data assimilation. In our vision only a probabilistic approach covering an ensemble of fast model realizations can help to build confidence in future predictions.

Instead of modeling an entire fractured system, many authors focused on injection into a single fracture (e.g. Mossop, 2001; Catalli et al., 2008; Baisch et al., 2010). This is justified by the fact that flow is often channelized in a single highly permeable fracture - instead of being homogeneously distributed in the medium. Relatively fast semi-analytical models, suitable for data assimilation schemes, already showed complex behavior in terms of couplings between stress perturbations due to pressure and temperature changes, permeability enhancement, and seismicity. In the same spirit, we focus on a single fracture injection model, but with the aim to assess the poro-elastic and thermo-elastic stress perturbations in the surrounding media. As a matter of fact, previous models were restricted in assessing the poro- and thermo-elastic stress perturbations solely at the fracture surface.

It is generally assumed that fault slip occurs when a Mohr-Coulomb stress criterion is reached. However, this approach, only considering stress changes, does not honor the frictional constitutive behavior of faults. Indeed, laboratory data and models based on rate and state friction show that the timing of a dynamic instability depends on both initial conditions and applied stress (Dieterich and Kilgore, 1996). Following a stress perturbation along a fault plane, an initial aseismic nucleation phase causes a delay in the start of a seismic instability. For a population of faults, such characteristic nucleation times become manifest in an Omori-type temporal decay of aftershocks after the stress perturbation induced by the mainshock. In our case the stress perturbation is the one imposed by the cold-water injection through the single fracture. This intrinsic rate dependence in earthquake nucleation will be also included in our approach in order to model the spatiotemporal pattern of the seismicity rate.

The present contribution is an extension of the work of Candela and Fokker (2017) where a semi-analytical approach following the nucleus-of-strain concept was presented. This time we introduce a fully analytical approach based on existing thermo-elastic theory to compute thermal stress changes.

\section{Pressure and temperature throughout and around a single fracture}

We employ an axisymmetric model for flow and temperature (Figure 1), based on the approach of Mossop (2001). Cold water (initial temperature $T_{w_{0}}=40^{\circ} \mathrm{C}$ ) is injected at constant rate into a horizontal permeable fracture surrounded by an impermeable hot medium (initial temperature $T_{R_{0}}=240^{\circ} \mathrm{C}$ ). Water is assumed to remain liquid and phase changes are neglected. 


\subsection{Pressure distribution}

In our flow model, we only consider the radial terms of the diffusion equation as:

$c_{D}\left(\frac{\partial^{2} p}{\partial r^{2}}+\frac{1}{r} \frac{\partial p}{\partial r}\right)-\frac{\partial p}{\partial t}=0$

where $r$ is the radial distance from the injection point. $c_{D}$ represents the fracture hydraulic diffusivity.

Boundary conditions are defined as:

$p(r \rightarrow \infty, t)=p_{0}$

$q_{r}\left(r=r_{b}, t \geq 0\right)=\frac{\dot{m}_{r_{b}}}{2 \pi r_{b} h}$

where $p_{0}$ is the initial pore pressure (constant in the complete domain), $r_{b}$ the borehole radius, $\dot{m}_{r_{b}}$ the mass injection rate at the borehole wall, $h$ the fracture thickness, and $q_{r}\left(r=r_{b}, t \geq 0\right)$ the radial component of the fluid flux at the borehole defined as constant. Introducing Darcy's law, equation (3) can be re-written as:

$\left.\lim _{r_{b} \rightarrow 0} r_{b} \frac{\partial p}{\partial r}\right|_{r=r_{b}}=-\frac{\dot{m}_{r_{b}} \eta}{2 \pi \rho_{w} k h}$

with $\eta$ and $\rho_{w}$ are respectively the water dynamic viscosity and density, and $k$ is fracture permeability. The solution of equation (1) subject to (2) and (4) gives the pore pressure distribution in the fracture as a function of radial distance from the injection point and time,

$p(r, t)=\frac{\dot{m}_{r_{b}} \eta}{4 \pi \rho_{w} k h} E_{1}\left(\frac{r^{2}}{4 c_{D} t}\right)+p_{0}$

(Theis, 1935), where $E_{1}$ is the exponential integral of the first kind (see Figure 2). Figure 2 presents the radial pressure distribution at different time steps of injection, and based on the model parameters listed in Table 1.

\subsection{Temperature distribution}

We assume that the flow of water through the fracture cools down the solid-rock component of the fracture by advection, and the surrounding rock (outside the fracture) by conduction. Following the approach of Mossop (2001) the problem is reduced to a radial flow heat exchanger and is a specific case of a general class of advection-diffusion problems. The main simplifying assumptions of the temperature model are:

* The fracture is thin enough (i) to assume thermal equilibrium between the water and solid-rock component of the fracture and (ii) to assume the temperature to be constant across the thickness of the fracture.

* Within the fracture, the radial thermal advection is supposed dominant and the radial thermal conduction is neglected.

* Within the rock outside the fracture, the normal conductive heat is supposed dominant and the radial conductive heat is neglected.

The temperature of the rock outside the fracture $T_{R}$ must satisfy the heat equation as:

$\nabla^{2} T_{R}-\frac{1}{\kappa} \frac{\partial T_{R}}{\partial t}=0$

where $\kappa$ is the rock thermal diffusivity. Energy conservation dictates the boundary conditions for solving equation (6): the heat flow from the rock to fracture (right side of 
equation (7)) must be balanced by the heat change in the fracture itself and the heat transported away throughout the fracture by the water (left side of equation (7)):

$\xi \frac{\partial T_{w}}{\partial t}+v \frac{\partial T_{w}}{\partial r}=\left.\frac{2 K}{h \phi \rho_{w} c_{w}} \frac{\partial T_{R}}{\partial z}\right|_{z= \pm h / 2}$

with $K$ the rock thermal conduction coefficient, $c_{w}$ the specific heat capacity, $v \rightarrow$ $\dot{m}_{r_{b}} /\left(2 \pi r h \rho_{w} \phi\right)$ the velocity of the radial flow inside the fracture (with $\phi$ the fracture porosity), and $\xi$ a dummy parameter close to unity quantifying the ratio between the total heat contained within the rock-fault and the total heat held in the pore water alone. Hence in equation (7) $T_{w}$ is equal to the temperature of the water and rock-fault since inside the fracture the water and solid-rock component are supposed at thermal equilibrium. The last boundary condition at the fracture interface imposes that the temperature of the wall rock, that is $T_{R}$ at the fracture interface, and $T_{w}$ should be in equilibrium:

$T_{w}=\left.T_{R}\right|_{z= \pm h / 2}$

Solving the heat equation (6) for the temperature perturbation $T_{R}$ in the rock outside the fracture with the boundary conditions [7] and [8] leads to:

$T_{R}(r, z, t)=T_{R_{0}}-\Delta T_{0} \operatorname{erfc}\left(\frac{a r^{2}+b|z|-\frac{h}{2}}{\sqrt{t-C r^{2}}}\right) H\left(t-C r^{2}\right) ; z \geq \frac{h}{2}$

$\Delta T_{0}=T_{R_{0}}-T_{W_{0}} \quad ; \quad a=\frac{\pi K}{\dot{m}_{r_{b}} c_{w} \sqrt{\kappa}} ; b=\frac{1}{2 \sqrt{\kappa}} ; C=\frac{\pi h \phi \xi \rho_{w}}{\dot{m}_{r_{b}}}$.

As already mentioned $T_{w}(r, t)$, temperature of the fluid and solid-rock in the fracture, is expressed as $T_{R}(r, z=h / 2, t)$. $H$ is for Heaviside function. The reader is referred to Appendix B of Mossop (2001) for the detailed derivation of the temperature perturbation (equation (9)). Figure 3 displays cross-sections of the temperature perturbation in a vertical plane passing through the borehole for the parameters listed in Table 1 and after different durations of injection.

\section{Poro-thermo-elastic stresses around the fracture}

An increase of pore pressure inside the fracture, due to the injection of water, causes the surrounding rock to be compressed. A decrease of the temperature inside and about the fracture causes the rock to contract. In poro-elasticity a dilation $e$ caused by an increase of the pore pressure $\Delta p$ is defined as $e=c_{m} \Delta p$ with $c_{m}$ the uniaxial compaction coefficient. In the case of thermo-elasticity, $c_{m}$ is replaced by $\left(\frac{1+v}{1-v}\right) \alpha$ (with $v$ the Poisson's ratio and $\alpha$ the coefficient of thermal expansion), and a contraction $e$ caused by a decrease of the temperature $\Delta T$ is thus defined as $e=\left(\frac{1+v}{1-v}\right) \alpha \Delta T$ (Geertsma, 1973).

We have adopted two approaches for deriving the stress field around the single fracture induced by poro- and thermo-elastic volumetric changes. The convention here is to use negative sign for compressive stresses.

\subsection{Nucleus-of-strain based: approach-A}

The first approach, referred to as approach-A in the sequel, is based on the nucleus-of-strain concept (Mindlin 1936, 1950, Sen, 1950). In line with our axisymmetric geometry we follow the approach developed by Geertsma (1973) and Segall (1992) for spatial integration of 
unitary nuclei over a disk-shaped reservoir. The pressure and temperature distributions are treated separately.

For each time step, the pressure distribution is divided into $n$ concentric hollow cylinders (Figure 4). The width of each hollow cylinder corresponds to the discrete radial steps, and the thickness of the hollow cylinders corresponds to the fracture thickness. Each hollow cylinder corresponds to a distribution of nuclei with identical pressure increase. The final solution is then given by the spatial integration of unitary nuclei of strain $\tau_{i j}{ }^{*}(r, z ; \varrho, \varsigma)$ over the radius $\varrho$ and thickness $\zeta$ of the perturbed zone as:

$\Delta \sigma_{i j}(r, z)=\iint_{\varsigma \varrho} e(\varrho, \varsigma) \tau_{i j}^{*}(r, z ; \varrho, \varsigma) d \varrho d \varsigma$

For the temperature distribution we follow the same approach as for the pressure, except that its depth-dependency requires an up-scaling step first. Indeed, for the sake of computational speed, the volumetric temperature distribution is vertically averaged to obtain a planar distribution. We have confirmed that performing the computation without up-scaling, that is considering a vertical array of discrete hollow cylinders, gives similar results for the stress distribution around the perturbed disk-shaped volume.

Instead of using the original single center of compression nucleus of strain developed by Mindlin (1936) for the stress induced by each nucleus, we used the newly developed influence function by Nikkhoo et al., (2017). This new solution extends the well-known Okada's inflation source solution (Okada, 1985, 1992) by addressing the numerical artefact and the geometrical limitation problem in his structure.

\subsection{Myklestad based: approach-B}

Myklestad (1942) derived equations (see Appendix A) for all the components of the stress tensor as induced by heating a semi-infinite cylinder to a constant temperature difference with respect to the ambient reservoir temperature using elliptical integrals in a radial coordinate system. The expressions for all stress components are built up from specific elliptic integrals. Before using Myklestad's approach, we had to change the use of the \pm -sign of his equation [M25], which was incorrect in the article (see Appendix A). To obtain the thermo-elastic stresses around a disk-shaped reservoir, that is the single fracture in our case, we subtracted the contributions of two cylinders, the second one translated vertically over the thickness $h$ of the zone affected by temperature changes (Figure 5). To derive the extent of the zone affected by temperature changes, the temperature distributions obtained with equation (9) were approximated by a cylindrically-shaped uniform distribution. For each time step, the thickness and radius of the cylinders correspond to the distances reached by the temperature front $-80^{\circ} \mathrm{C}$ respectively at the injection point and the tip of the fracture. The averaged uniform temperature perturbation is approximated as $T_{R}=-140^{\circ} \mathrm{C}$ (see Figure 3).

Figures 6 and 7 present examples of stress fields around the single fracture computed with model parameters listed in Table 1 for our both approaches. Tensor of stress changes were first translated to Cartesian coordinates using the standard cylindrical coordinate transformation (Fjaer et al., 2008). Both approaches give similar thermo-elastic stress distributions. Beyond 50m away from the single fracture, the induced poro-elastic stresses 
are negligible compared to the induced thermo-elastic stresses. In the following, therefore, the Coulomb stressing rate and the associated seismicity rate will be based on the thermoelastic stresses only.

\section{Coulomb stress}

We consider faults uniformly distributed around the single fracture. In other words, each location around the single fracture can potentially host a fault. For this particular example we consider only one fault family, that is all the faults are normal faults striking N-S and dipping $60^{\circ}$ toward west (see Figure 1). This setting results in a unit normal vector to the fault $\widehat{\boldsymbol{n}}=[-0.866,0,0.5]^{T}$ and a unit slip vector $\widehat{\boldsymbol{s}}=[-0.5,0,-0.866]^{T}$.

Before determining the Coulomb stress, one must compute the shear and normal tractions. This can be done combining the stress tensor with the unit slip and normal vectors (Jaeger et al., 2007). Finally the Coulomb stress changes reads:

$\Delta S=\Delta \tau_{s}+f\left(\Delta \sigma_{n}\right)$

with $\Delta \tau_{s}$ and $\Delta \sigma_{n}$ the shear and normal tractions changes computed on the assumed fault plane, $f$ the fault friction coefficient.

\section{Seismicity rate}

We intend to model the seismicity rate induced by the thermo-elastic stress changes. The traditional Coulomb failure model predicts that whenever the Coulomb stress reaches a threshold value, an earthquake is generated. Assuming a population of faults on which the pre-stresses are uniformly distributed up to the threshold value, the Coulomb failure model leads to a direct proportionality between the seismicity rate and the Coulomb stress rate. During any arbitrary stressing history, as soon as the Coulomb stress starts to decrease, the Coulomb failure model predicts an instantaneous shut-down of the seismicity. This prediction is not in agreement with the observed seismicity, which generally shows a gradual decay following the onset of Coulomb stress decrease.

The Coulomb failure model does not honor the frictional constitutive behavior of faults. Laboratory data show that the timing of dynamic instability depends on initial conditions, fault properties and applied stress (Dieterich and Kilgore, 1996). The rate-and-state friction laws have been established in order to reproduce these laboratory observations (see Marone, 1998 for a review). More specifically, the rate-and-state friction laws reproduce the fact that the onset of frictional sliding is a non-instantaneous time-dependent process (as opposed to the instantaneity assumption of the Coulomb model), which introduces a timedependent failure mechanism for the generation of earthquakes. Now assuming a population of faults following a rate-and-state frictional behavior, and where the time-tofailure of the nucleation spots along the faults is uniformly distributed, Dieterich (1994) derived the following seismicity rate model:

$R_{D}=\frac{r_{0}}{\gamma \dot{S}_{0}}$ where $\frac{d \gamma}{d t}=\frac{1}{A \bar{\sigma}}\left[1-\gamma \frac{d S}{d t}\right]$

where $R_{D}$ is the seismicity rate, $\gamma$ is a state variable, $S$ is the Coulomb stress and $\bar{\sigma}$ the background effective normal stress. The constant $r_{0}$ is the steady-state background 
seismicity rate at the reference stressing rate $\dot{S}_{0} . A$ is a dimensionless fault constitutive parameter.

Segall and Lu (2015) reformulated this seismicity rate equation to eliminate the state variable $\gamma$. They defined a normalized seismicity rate, relative to the background rate, as:

$R=\frac{R_{D}}{r_{0}}$

The differential equation for $R$, derived from equations (12) and (13), is:

$\frac{d R}{d t}=\frac{R}{t_{a}}\left[\frac{\dot{S}}{\dot{S}_{0}}-R\right]$

where $t_{a}=A \sigma_{n}^{\prime} / \dot{S}_{0}$ is the characteristic time delay for the earthquake nucleation process, which also corresponds to the time scale of decay of the aftershock rate following a main shock back to the background rate.

The rate of seismicity $R$ can be viewed as a statistical representation of the rate of earthquakes of a given magnitude, such that $R=100$ corresponds to a hundredfold increase in the rate of earthquakes of a given magnitude.

Before computing the rate of seismicity $R$ with equation (14), one needs to calculate the Coulomb stressing rate $\dot{\tau}$. This is realized by following an approach similar to Chang and Segall (2016). From equation (11), we obtained the Coulomb stress at discrete time steps. Then we used a smoothing interpolant in order to estimate the Coulomb stress history at arbitrary time steps. This interpolation step needs to be performed with caution and we used a spline form (using pchip in MATLAB) that allows to avoid ringing behavior near derivative singularities. Finally, one can differentiate the Coulomb stress interpolant (using fnder in MATLAB).

After calculation of the Coulomb stressing rate $\dot{\tau}$, we integrate the ordinary differential equation (14) using ode45 in MATLAB with relative tolerance of $1 \mathrm{e}-6$ and very small absolute tolerance. Figures 8 to 10 display the spatio-temporal patterns of the seismicity rates solely induced by thermo-elastic stressing and following the Myklestad-based approach (section 3.2).

As a reference scenario, for friction parameters we assume a nominal friction of $f=0.6$, and we take $a=0.001$ as representative of friction experiments (Marone, 1998). We consider a background effective normal stress $\bar{\sigma}=40 \mathrm{MPa}$, and assume a background stressing rate $\dot{\tau}_{0}=0.8 \times 10^{-3} \mathrm{MPa} /$ years such that a typical $0.8 \mathrm{MPa}$ stress drop accumulates in 1000 years. Combining these parameters values, this leads to a characteristic decay time $t_{a}=50$ years. We also consider two alternative scenarios. In the first, the friction is reduced to 0.1 following the assumption that the potential seismic faults are smeared by minerals with low friction coefficients (see e.g. Lockner et al., 2011; Di Toro et al., 2011). The contribution of $\Delta \sigma_{n}$ in the fault destabilization is then reduced (see equation 11). The second alternative employed a lower background effective normal stress $\bar{\sigma}=$ $10 \mathrm{MPa}$ representative of over-pressurized faults, also implying a shorter characteristic time delay $t_{a}$.

For the particular fault family set used, the increase of the seismicity rate $R$ can be as much as ten thousand $\left(\log _{10} R=4\right)$ at $100 \mathrm{~m}$ from the single fracture (Figures 8 to 10 ). With a 
decrease of the fault friction the seismicity rate is even higher, as the shear stress contribution is magnified. With a shortening of $t_{a}$, by the reduction of the effective normal stress, the increase of the seismicity rate is even faster.

\section{Discussions and Conclusions}

The purpose of this paper was first to depict the details of the fast forward modelling steps rather than presenting sensitivity analysis on the model outcomes varying the model parameters. We detailed two independent fast routes for modelling induced poro- thermoelastic stresses around a single fracture. Figures 6 and 7 reveal that thermo-elastic effects are dominant compared to poro-elastic effects at distances beyond $50 \mathrm{~m}$ from the fracture. The same injection model could be also adapted to thicker high-permeability streaks like fracture corridors; in this case the volumetric strain induced by injection might lead to more significant poro-elastic stressing. It is interesting to see that even with a very thin fracture, the thermal disturbance can induce a more than thousand-fold increase in the rate of seismicity $\left(\log _{10} R>3\right.$ ) at distances up to $200 \mathrm{~m}$ from the single fracture (see Figure 8 ). Of course, different families of faults with different orientations would result in different spatio-temporal patterns of seismicity rate. We have also demonstrated that fault zone frictional properties and in-situ stress properties are essential in estimating seismicity rates.

The next step of our approach would be to integrate the present forward models in a data assimilation scheme. Both forward models and model parameters should be considered in order to rank their performance. The exercise here consists in confronting model outcomes with data in order to refine knowledge about the governing physical processes and the driving input model parameters. The spatio-temporal distribution of induced microearthquakes is the natural candidate for the data to be assimilated. At this stage, simplifying assumptions of the model could be tested, and one could imagine a step-wise complexification of the forward modeling in order to improve the match with the data. For example one should be able to assess whether or not full temperature $\leftrightarrow$ pressure $\leftrightarrow$ stress coupling should be integrated in the forward model in order to match the data and process of interest. Refining the model parameters of the Dieterich (1994) model would be also a purpose of the data assimilation scheme (Catalli, 2008). The limitations of the Dieterich's model, as disregarding the source-to-source stress interactions, could be also tested by data assimilation. Finally, one could make the connection to more micro-physics based friction models (Niemeyer and Spiers, 2007; Chen and Spiers, 2016).

We presented fast analytical routes suitable for data assimilation in order to model and predict seismicity rates in a fractured geothermal system. Our approach combining porothermo-elastic stressing and fault constitutive behavior has been tailored to model seismicity rates around a single fracture in a geothermal field. Previous fast models were often restricted in assessing the poro- and thermo-elastic stress perturbations and seismicity solely at the fracture surface. Clearly both approaches are complementary and one could easily imagine combining both as a next forward modeling. Interestingly, we showed that even with a very thin single fracture, thermal-stressing rates can induce an increase in the rate of seismicity of more than hundredfold at distances up to $200 \mathrm{~m}$ from the fracture. By predicting the spatio-temporal evolution of the locations of 
increase/decrease of seismicity rate one should be able to optimize geothermal systems while keeping seismicity at a relatively low magnitude, thus avoiding risks of large earthquakes.

\section{Acknowledgements}

This paper is part of a project that received funding by the European Union's Horizon 2020 research and innovation programme under grant agreement number 691728 (DESTRESS). This work was also part of the Geocap project (Geothermal Capacity Building Program Indonesia - Netherlands), and international collaboration between Indonesian and Duch partners (https ://www.geoca p.nl/), funded by the Ministry of Foreign Affairs of the Netherlands.

Table 1. Model parameters

\begin{tabular}{|c|c|}
\hline \multicolumn{2}{|c|}{ Pressure \& Temperature model } \\
\hline$T_{w_{0}}$ & $40^{\circ} \mathrm{C}$ \\
\hline$T_{R_{0}}$ & $240^{\circ} \mathrm{C}$ \\
\hline$r_{b}$ & $0.1 \mathrm{~m}$ \\
\hline$\dot{m}_{r_{b}}$ & $10 \mathrm{~kg} \mathrm{~s}^{-1}$ \\
\hline$c_{D}$ & $1000 \mathrm{~m}^{2} \mathrm{~s}^{-1}$ \\
\hline$h$ & $0.001 \mathrm{~m}$ \\
\hline$\eta$ & $10^{-3} \mathrm{~kg} \mathrm{~m}^{-1} \mathrm{~s}^{-1}$ \\
\hline$k$ & $10^{-8} m^{2}$ \\
\hline$\rho_{w}$ & $1000 \mathrm{~kg} \mathrm{~m}^{-3}$ \\
\hline$\kappa$ & $10^{-6} \mathrm{~m}^{2} \mathrm{~s}^{-1}$ \\
\hline$K$ & 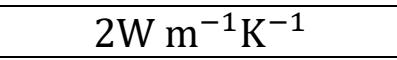 \\
\hline$\phi$ & 1.0 \\
\hline$c_{w}$ & $4200 \mathrm{~J} \mathrm{~kg}^{-1} \mathrm{~K}^{-1}$ \\
\hline$\xi$ & 1.0 \\
\hline \multicolumn{2}{|c|}{ Mechanical model } \\
\hline$C_{m}$ (pressure) & $1.5 \times 10^{-5} \mathrm{bar}^{-1}$ \\
\hline$C_{m}$ (temperature) & $1.7 \times 10^{-5} \mathrm{~K}^{-1}$ \\
\hline$\mu$ & $0.3 \times 10^{11} \mathrm{~Pa}$ \\
\hline$\lambda$ & $0.3 \times 10^{11} \mathrm{~Pa}$ \\
\hline$f$ & $0.6 \& 0.1$ \\
\hline$c_{D}$ & $1000 m^{2} s^{-1}$ \\
\hline $\mathrm{a}$ & 0.001 \\
\hline $\bar{\sigma}$ & $40 \& 10 \mathrm{MPa}$ \\
\hline$\dot{\tau}_{0}$ & $0.8 \times 10^{-3} \mathrm{MPa} /$ years \\
\hline
\end{tabular}




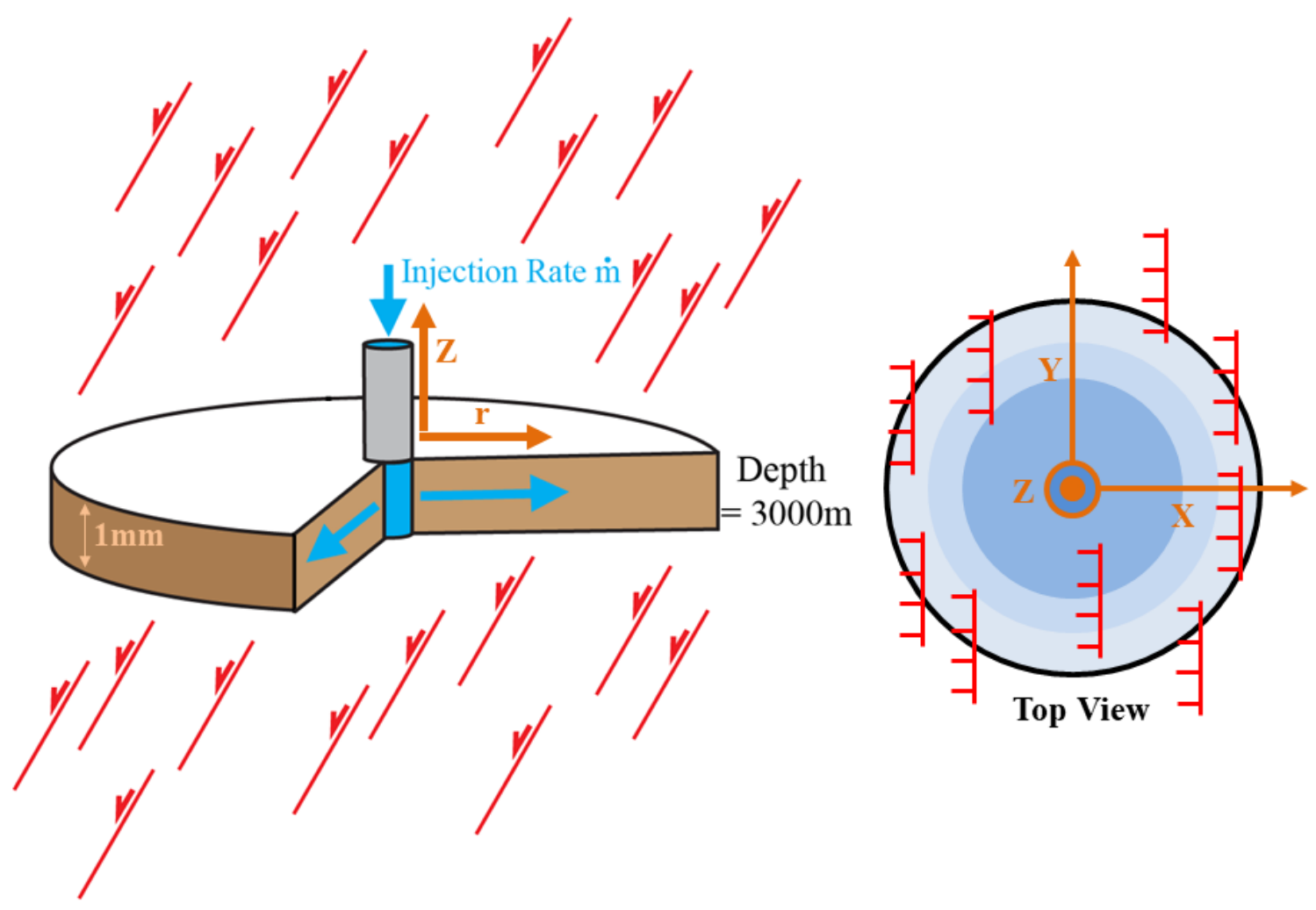

Fig. 1. Model geometry. The origin is at the center of the borehole in the middle of the single fracture (depth $=3000 \mathrm{~m}$, thickness $=1 \mathrm{~mm}$ ). Potential normal faults (in red) are dipping to the west. 


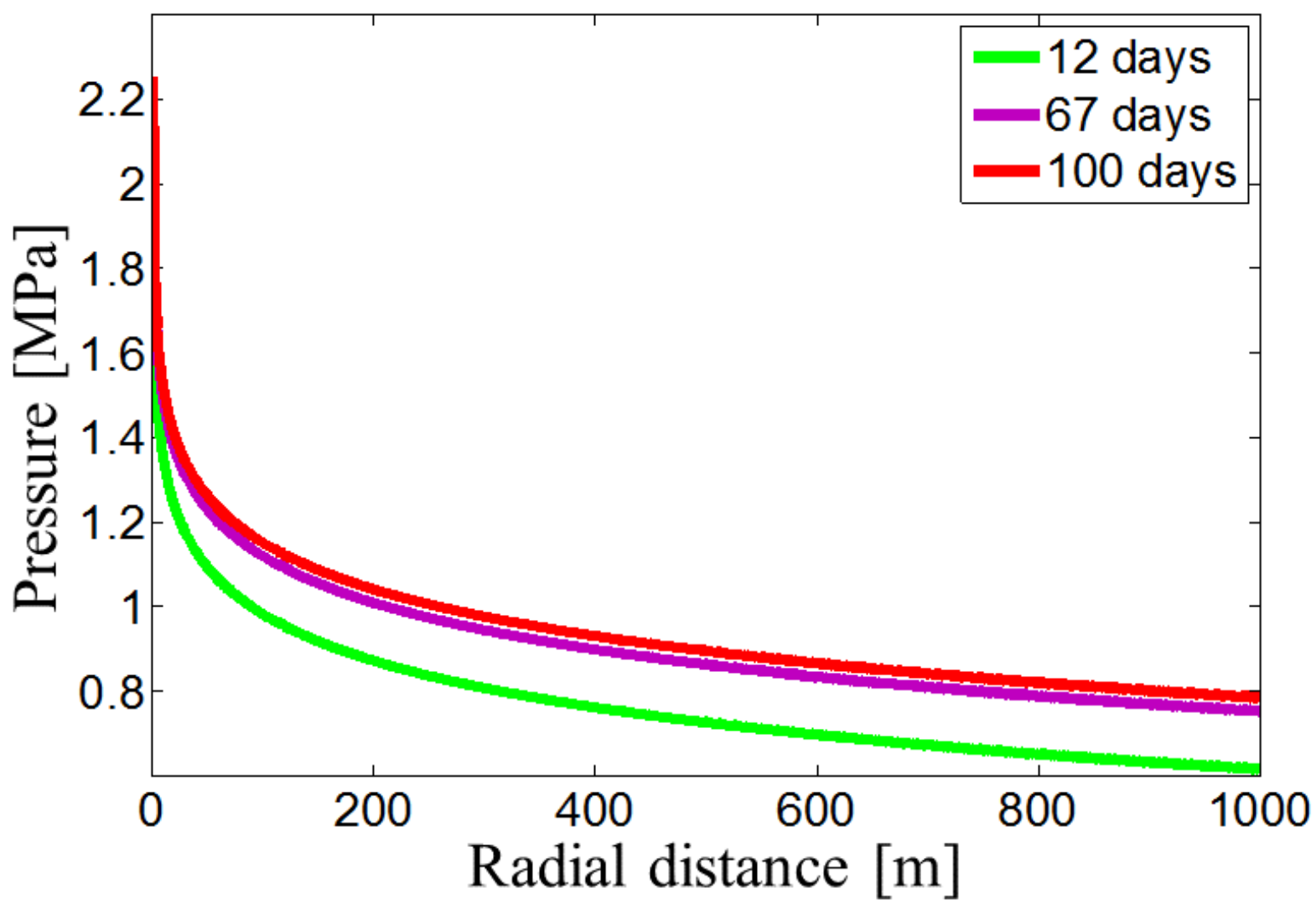

Fig. 2 Pressure distributions for three different time steps: after 12, 67 and 100 days of injection. 

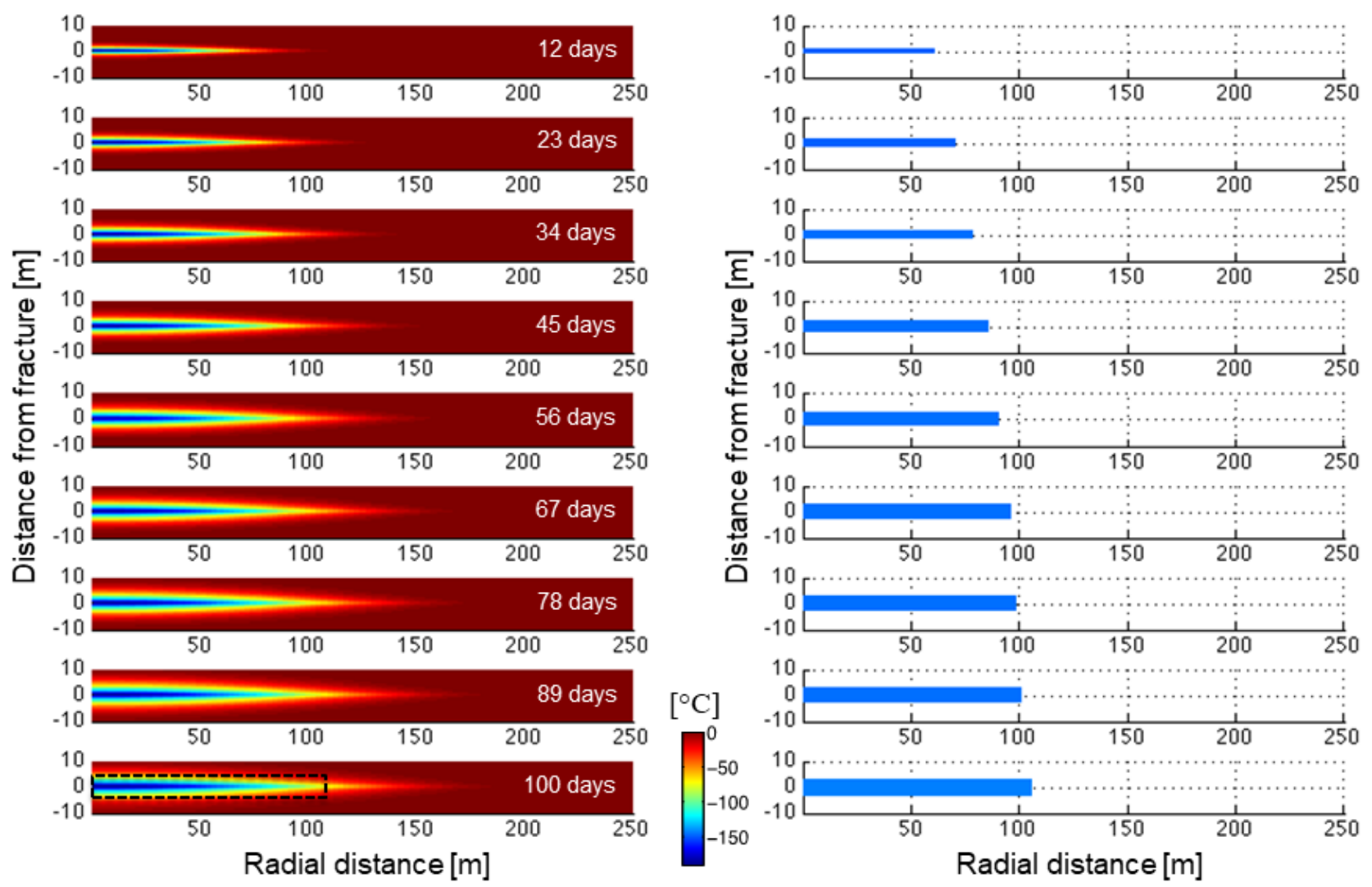

Fig. 3 Temperature distributions for different days of injections. Left side: temperature distributions obtained with equation 9 and used for the approach-A. The dashed black contour on the bottom panel underlines the cylindrical approximations used for the approach B (Right side). In order to derive the thickness and radius of the cylinders we picked the distance reached by the temperature front $-80^{\circ} \mathrm{C}$ respectively at the injection point and the tip of the fracture. Right side: Cylindrical approximations of the left-side temperature distributions used for the approach-B. The uniform temperature perturbation $T_{R}$ of each cylindrical field is $-140^{\circ} \mathrm{C}$. 


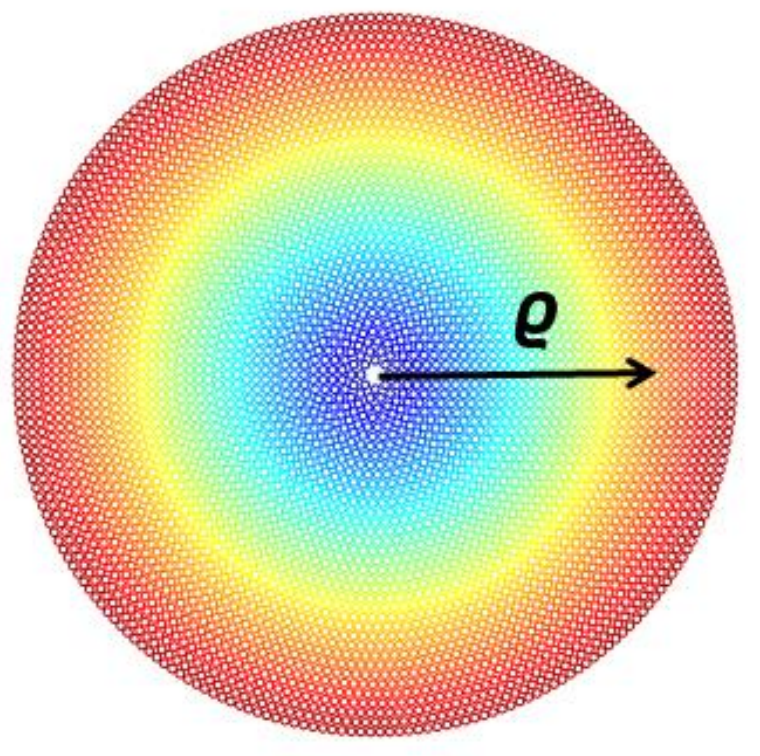

Fig. 4 Concentric hollow cylinders of nuclei. The color scale marks the distance of each row from the origin (center of the borehole - middle of the single fracture). 


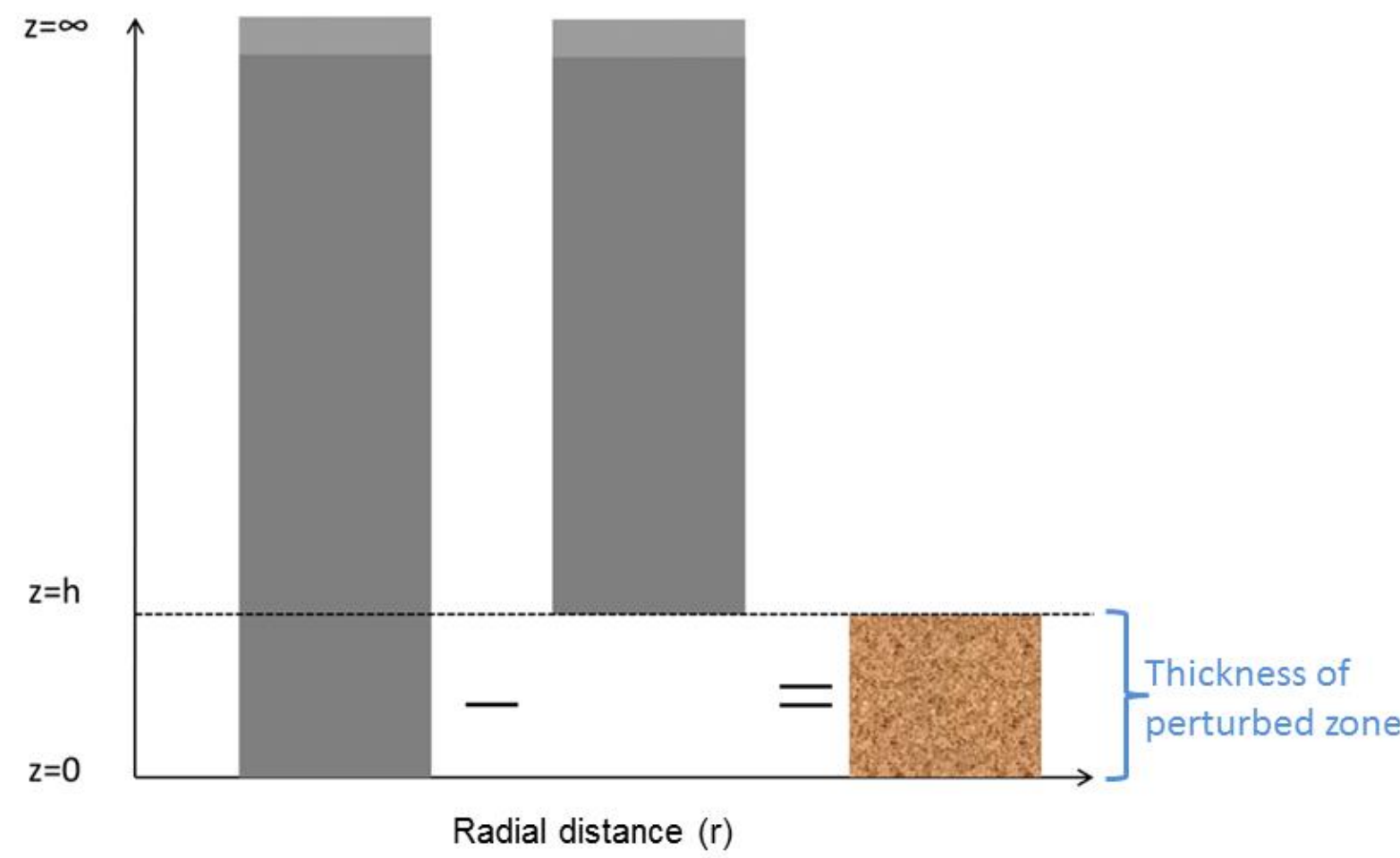

Fig. 5 Set-up of the thermo-elastic approach B for stress changes around the single fracture based on semi-infinite cylinders as proposed by Myklestad (1942). See also Appendix A. 


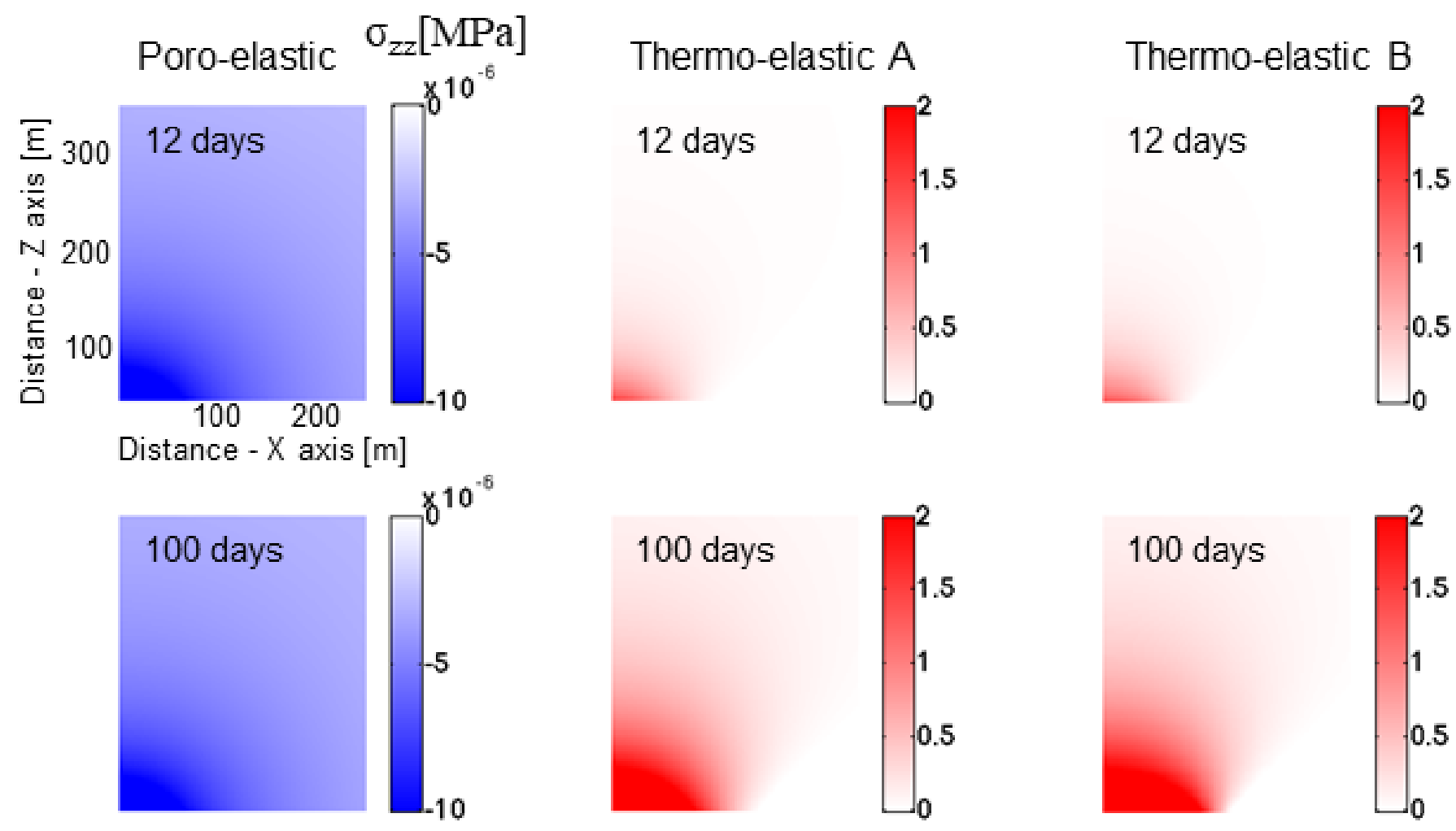

Fig. 6 Poro- and thermo- elastic $\sigma_{z z}$ stress fields around the single fracture. $\mathrm{X}$ axis is the E-W direction perpendicular to fault strike, and $Z$ axis is the vertical direction on top of the single fracture (see Figure 1). Thermo-elastic $A$ and $B$ stand for thermo-elastic stresses computed via respectively approach $\mathrm{A}$ and $\mathrm{B}$. 


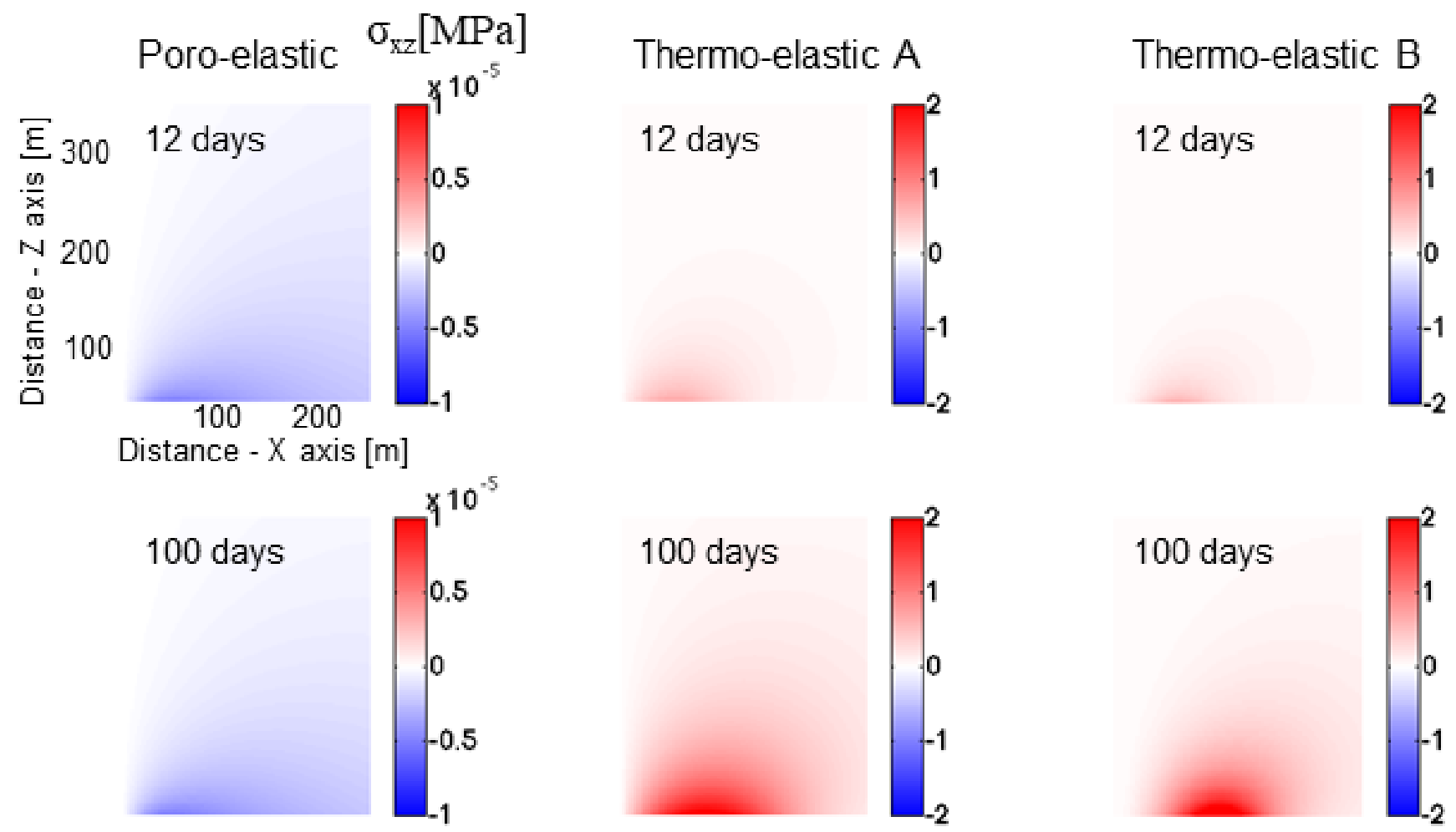

Fig. 7 Poro- and thermo- elastic $\sigma_{x z}$ stress fields around the single fracture. $\mathrm{X}$ axis is the $\mathrm{E}-\mathrm{W}$ direction perpendicular to fault strike, and $Z$ axis is the vertical direction on top of the single fracture (see Figure 1). Thermo-elastic $A$ and $B$ stand for thermo-elastic stresses computed via respectively approach $A$ and $B$. 


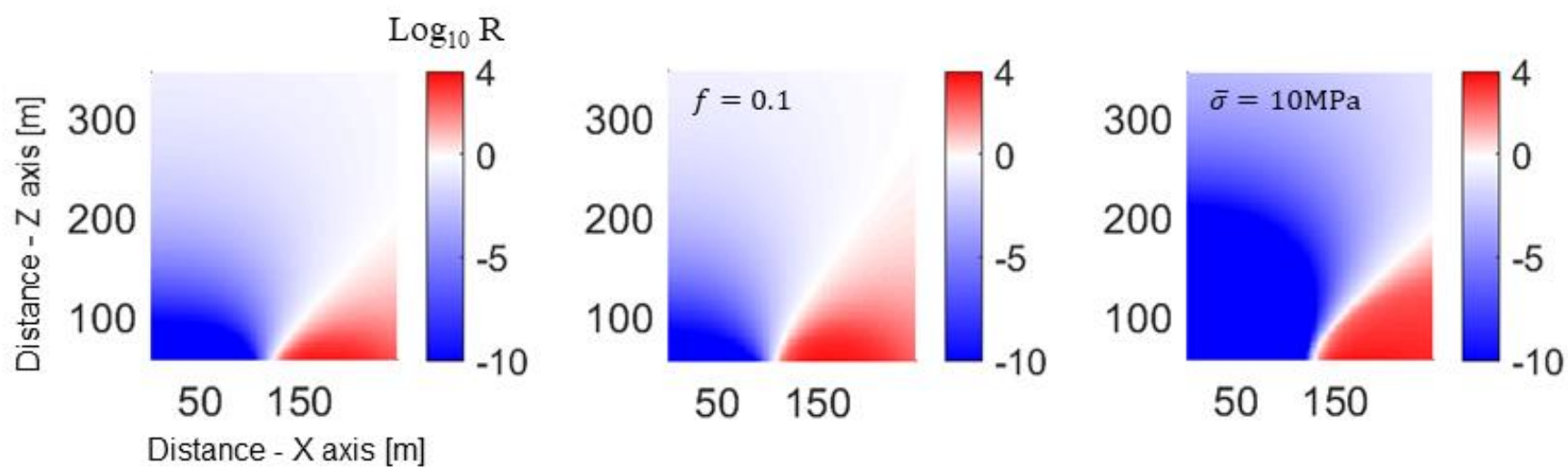

Fig. 8 Spatial distribution of the seismicity rate $\left(\log _{10} R\right)$ induced by thermo- elastic stressing after 100 days of injection around the single-fracture. $X$ axis is the E-W direction perpendicular to fault strike, and $\mathrm{Z}$ axis is the vertical direction on top of the single fracture (see Figure 1). Left: reference case with friction $f=0.6$ and $\bar{\sigma}=40 \mathrm{MPa}$; center: $f=0.1$ and $\bar{\sigma}$ $=40 \mathrm{MPa}$; right: $f=0.6$ and $\bar{\sigma}=10 \mathrm{MPa}$. 


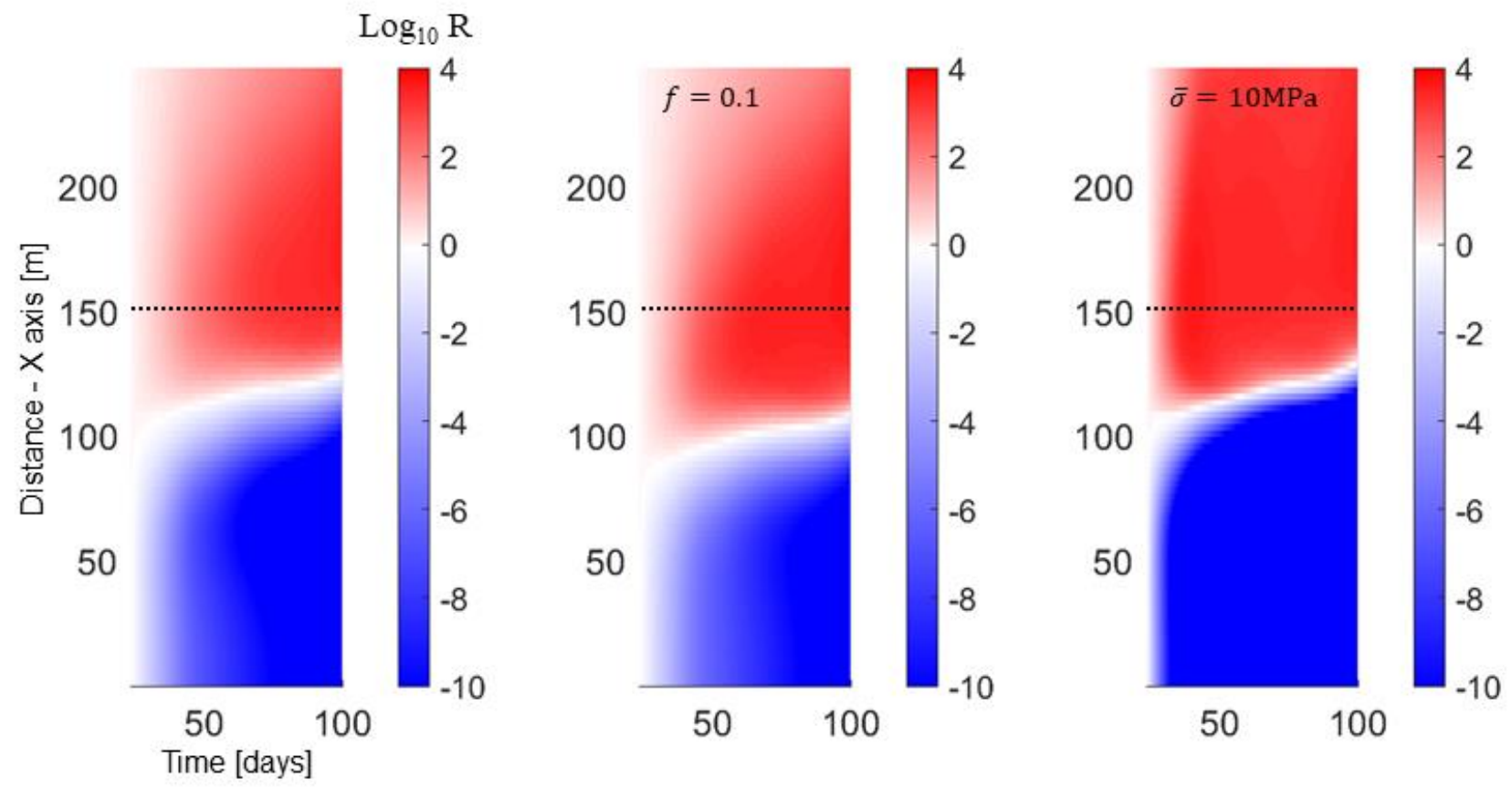

Fig. 9 Spatio-temporal distribution of the seismicity rate $\left(\log _{10} R\right)$ induced by thermo- elastic stressing along the $X$ axis ( $E-W$ direction perpendicular to fault strike in Figure 1 ) and at a distance $Z=60 \mathrm{~m}$ on top of the single fracture. Left: reference case with friction $f=0.6$ and $\bar{\sigma}=40 \mathrm{MPa}$; center: $f=0.1$ and $\bar{\sigma}=40 \mathrm{MPa}$; right: $f=0.6$ and $\bar{\sigma}=10 \mathrm{MPa}$. The horizontal black dashed lines marks the chosen location (i.e. the distance along the $X$ axis $=150 \mathrm{~m}$ ) for the slices presented in Fig. 10. 


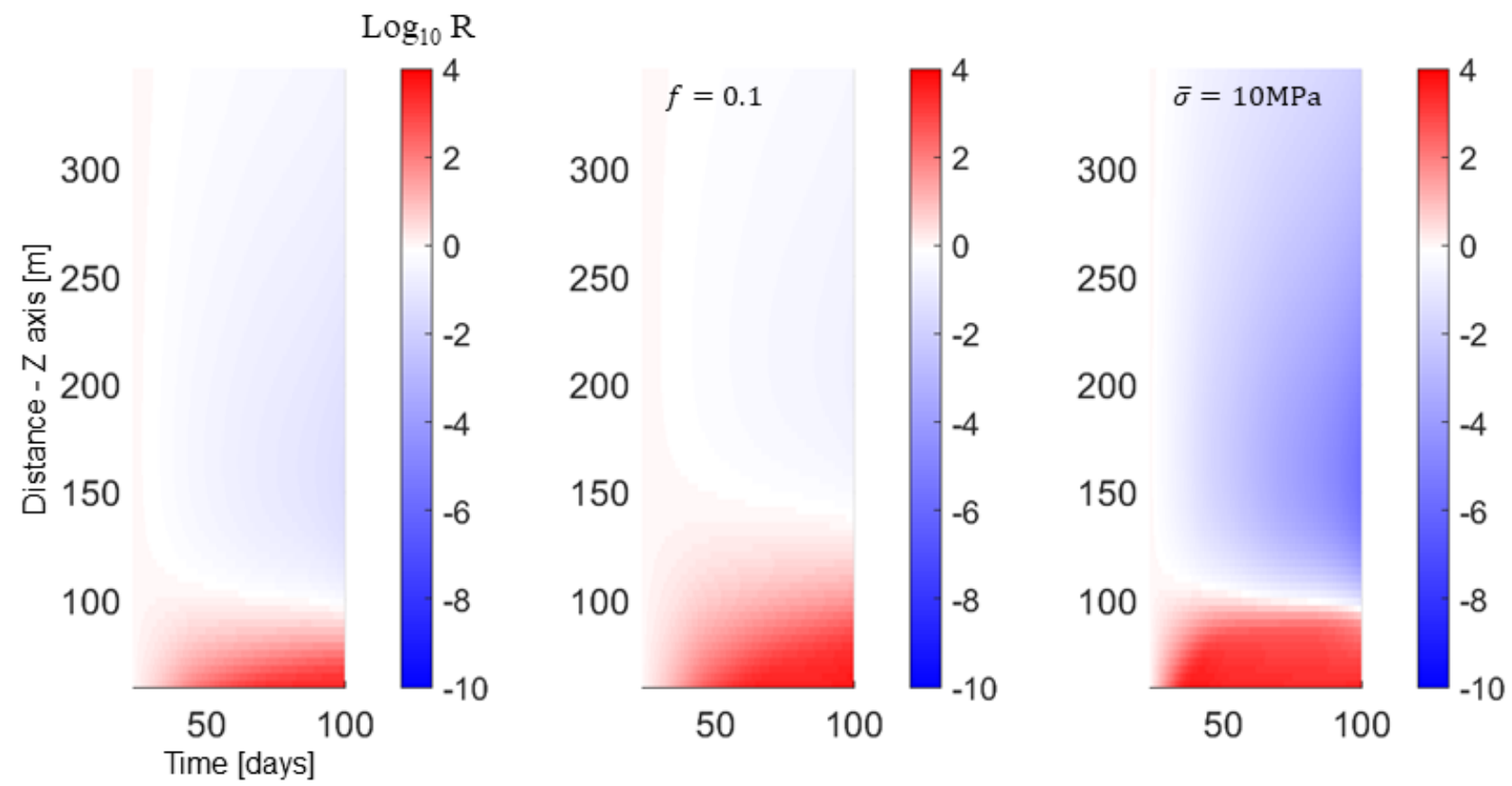

Fig. 10 Spatio-temporal distribution of the seismicity rate $\left(\log _{10} R\right)$ induced by thermoelastic stressing along the $Z$ axis (on top of the fracture) and at a distance along the $X$ axis = $150 \mathrm{~m}$ (see Figure 9). Left: reference case with friction $f=0.6$ and $\bar{\sigma}=40 \mathrm{MPa}$; center: $f=0.1$ and $\bar{\sigma}=40 \mathrm{MPa}$; right: $f=0.6$ and $\bar{\sigma}=10 \mathrm{MPa}$. 


\section{Appendix A Analytical solutions for the thermo-elastic stresses in and around a semi- infinite cylinder}

Myklestad (1942) has proposed equations for the shear stress and radial, tangential and vertical normal stress in and around a relatively cold semi-infinite cylinder in a relatively hot, isotropic, infinite solid. This analytical solution distinguishes between several regions in and around the cylinder. For convenience, radius $r$ and depth $z$ values are normalized to the radius of the cylinder, giving $p=\frac{r}{a}$ and $n=\frac{z}{a}$, where $\mathrm{a}$ is the radius of the cylinder. The equations are indicated by the letter $M$ followed by a number, to illustrate to which equation in Myklestads paper they are linked. Figure 5 shows the set-up of a semi-infinite cylinder with the different locations corresponding to the set of equations presented.

The equations by Myklestad (1942) make use of elliptic integrals, which are defined as:

$\bar{K}=\int_{0}^{\frac{\pi}{2}} \frac{d \theta}{\sqrt{1-k^{2} \sin ^{2} \theta}}$

$\bar{E}=\int_{0}^{\frac{\pi}{2}} \sqrt{1-k^{2} \sin ^{2} \theta} d \theta$ modulus $\mathrm{k}$

$\bar{K}^{\prime}=\int_{0}^{\frac{\pi}{2}} \frac{d \theta}{\sqrt{1-k^{\prime 2} \sin ^{2} \theta}}$

$\bar{E}^{\prime}=\int_{0}^{\frac{\pi}{2}} \sqrt{1-k^{\prime 2} \sin ^{2} \theta} d \theta$

$\bar{K}\left(\varphi^{\prime}, k^{\prime}\right)=\int_{0}^{\varphi \prime} \frac{d \theta}{\sqrt{1-k^{\prime 2} \sin ^{2} \theta}}$

$\bar{E}\left(\varphi^{\prime}, k^{\prime}\right)=\int_{0}^{\varphi \prime} \sqrt{1-k^{\prime 2} \sin ^{2} \theta} d \theta$ incomplete elliptic integral of the second kind, modulus $\mathrm{k}^{\prime}$

The modulus used to evaluate the elliptic integrals differs for the shear and vertical normal stress, and the radial and tangential normal stress. The moduli for the elliptic integrals are described as:

$k=\sqrt{\frac{a^{2}+\lambda_{2}}{a^{2}+\lambda_{1}}}$ for the vertical stress change $\Delta \sigma_{\mathrm{z}}$ and the shear stress change $\Delta \tau_{\mathrm{rz}}$

$\lambda_{1}=\frac{1}{2}\left[r^{2}+z^{2}-a^{2}+\sqrt{\left(r^{2}+z^{2}-a^{2}\right)^{2}+4 a^{2} z^{2}}\right]$

$\lambda_{2}=\frac{1}{2}\left[r^{2}+z^{2}-a^{2}-\sqrt{\left(r^{2}+z^{2}-a^{2}\right)^{2}+4 a^{2} z^{2}}\right]$

$k=\sqrt{\frac{4 p}{(p+1)^{2}+n^{2}}}$

$k^{\prime}=\sqrt{1-k^{2}}$

for the radial and tangential normal stress change $\Delta \sigma_{r}, \Delta \sigma_{t}$

Similarly, phase $\phi^{\prime}$ for the incomplete elliptic integrals, differs for the radial and tangential stress on the one hand, and the vertical and shear stresses on the other hand. They are defined as:

$\sin ^{2} \varphi^{\prime}=\frac{-\frac{1}{2}\left(r^{2}+z^{2}-a^{2}-\sqrt{r^{2}+z^{2}-a^{2}+4 a^{2} z^{2}}\right)}{k^{\prime 2} a^{2}} \quad$ for the vertical and shear stress change

$\sin ^{2} \varphi^{\prime}=\frac{n^{2}}{(p-1)^{2}+n^{2}} \quad$ for the radial and tangential stress change

Using the above description of elliptic integrals, the equations used for the contribution of a semi-infinite cylinder can be obtained.

\section{Shear stresses}


$\Delta \tau_{r Z}=\frac{E \alpha_{T} \Delta T}{1-v} \cdot \frac{1}{\pi} \sqrt{\frac{1}{p k}}(\bar{K}-\bar{E})$

$\Delta \tau_{r \theta}=0$ for all $r, z[M 17]$

for all r, z [M17]

\section{Vertical stress}

$\Delta \sigma_{z}= \pm \frac{E \alpha_{T} \Delta T}{1-v}\left[\frac{K}{\pi}\left(\bar{E}\left(\varphi^{\prime}, k^{\prime}\right)-\frac{\bar{K}\left(\varphi^{\prime}, k^{\prime}\right) E^{\prime}}{K^{\prime}}-\sqrt{\frac{(1-p k)(p-k)}{p}}\right)+\frac{1}{2} \frac{\bar{K}\left(\varphi^{\prime}, k^{\prime}\right)}{K^{\prime}}\right]$

where the minus sign is used for negative $z$-values, and the plus sign for positive $z$-values [M18]. This equation is valid for $r>a$ or $r \leq a \& z<0$.

$\Delta \sigma_{z}= \pm\left(\frac{E \alpha_{T} \Delta T}{1-v}\left[\frac{K}{\pi}\left(\bar{E}\left(\varphi^{\prime}, k^{\prime}\right)-\frac{\bar{K}\left(\varphi^{\prime}, k^{\prime}\right) E^{\prime}}{K^{\prime}}-\sqrt{\frac{(1-p k)(p-k)}{p}}\right)+\frac{1}{2} \frac{\bar{K}\left(\varphi^{\prime}, k^{\prime}\right)}{K^{\prime}}\right]-\frac{E \alpha \Delta T}{1-v}\right)$

for $r \leq a, r \neq 0$ and $z \geq 0[M 18]$.

$\Delta \sigma_{z}=-\frac{1}{2} \frac{E \alpha_{T} \Delta T}{1-v}\left[1+\frac{n}{\sqrt{1+n^{2}}}\right]$ for $\mathrm{r}=0, \mathrm{z} \geq 0$ [M19]

\section{$\underline{\text { Radial stress }}$}

$\Delta \sigma_{r}=-\frac{E \alpha_{T} \Delta T}{1-v} \frac{1}{4 \pi p^{2}}\left[\pi+\frac{n}{\sqrt{(p+1)^{2}+n^{2}}}\left[\left((p+1)^{2}+n^{2}\right)(K-E)-\left(p^{2}+2 p-1\right) K\right] \pm\left(p^{2}+\right.\right.$

1) $\left.\left[K \bar{E}\left(\varphi^{\prime}, k^{\prime}\right)-\frac{K E^{\prime} \bar{K}\left(\varphi^{\prime}, k^{\prime}\right)}{K^{\prime}}+\frac{\pi}{2} \frac{\bar{K}\left(\varphi^{\prime}, k^{\prime}\right)}{K^{\prime}}\right]\right]$

for all exterior points at $r>a$ [M24]. Again, for negative $z$-values the last term is positive, whereas for positive $z$-values, the last term is negative.

$\Delta \sigma_{r}=\frac{E \alpha_{T} \Delta T}{1-v} \frac{1}{4 \pi}\left[\pi-\frac{n}{p^{2} \sqrt{(p+1)^{2}+n^{2}}}\left[\left((p+1)^{2}+n^{2}\right)(K-E)-\left(p^{2}+2 p-1\right) K\right]-\right.$

$\left.\frac{p^{2}+1}{p^{2}}\left[K \bar{E}\left(\varphi^{\prime}, k^{\prime}\right)-\frac{K E^{\prime} \bar{K}\left(\varphi^{\prime}, k^{\prime}\right)}{K^{\prime}}+\frac{\pi}{2} \frac{\bar{K}\left(\varphi^{\prime}, k^{\prime}\right)}{K^{\prime}}\right]\right]$

for all exterior points $r \leq a, r \neq 0$ and $z<0[M 24 a]$.

$\Delta \sigma_{r}=-\frac{E \alpha_{T} \Delta T}{1-v} \frac{1}{4 \pi}\left[3 \pi+\frac{n}{p^{2} \sqrt{(p+1)^{2}+n^{2}}}\left[\left((p+1)^{2}+n^{2}\right)(K-E)-\left(p^{2}+2 p-1\right) K\right]-\right.$

$\left.\frac{p^{2}+1}{p^{2}}\left[K \bar{E}\left(\varphi^{\prime}, k^{\prime}\right)-\frac{K E^{\prime} \bar{K}\left(\varphi^{\prime}, k^{\prime}\right)}{K^{\prime}}+\frac{\pi}{2} \frac{\bar{K}\left(\varphi^{\prime}, k^{\prime}\right)}{K^{\prime}}\right]\right]$

for all interior points $r \leq a, r \neq 0$ and $z \geq 0$ [M24b].

$\Delta \sigma_{r}=\frac{1}{4} \frac{E \alpha_{T} \Delta T}{1-v}\left[1+\frac{n}{\sqrt{1+n^{2}}}\right]$

for all points along the $z$-axis with $r=0$ and $z<0$ [M26a].

$\Delta \sigma_{r}=-\frac{1}{4} \frac{E \alpha_{T} \Delta T}{1-v}\left[3-\frac{n}{\sqrt{1+n^{2}}}\right]$

for all points along the $z$-axis with $r=0$ and $z \geq 0$ [M26].

\section{$\underline{\text { Tangential stresses }}$}




$$
\begin{aligned}
& \Delta \sigma_{t}=\frac{E \alpha_{T} \Delta T}{1-v} \frac{1}{4 \pi p^{2}}\left[\pi+\frac{n}{\sqrt{(p+1)^{2}+n^{2}}}\left[\left((p+1)^{2}+n^{2}\right)(K-E)+(p-1)^{2} K\right] \pm\left(p^{2}-\right.\right. \\
& \text { 1) } \left.\left[K \bar{E}\left(\varphi^{\prime}, k^{\prime}\right)-\frac{K E^{\prime} \bar{K}\left(\varphi^{\prime}, k^{\prime}\right)}{K^{\prime}}+\frac{\pi}{2} \frac{\bar{K}\left(\varphi^{\prime}, k^{\prime}\right)}{K^{\prime}}\right]\right]
\end{aligned}
$$

for all exterior points at $r>a$ [M25]. For negative $z$-values the last term is negative, whereas for positive z-values, the last term is positive. Note that for equation [M25], the \pm -sign is used in a different way, than for the rest of the equations. Myklestad (1942) does not indicate this, but the model-checks we applied have pointed out that it is the case.

$$
\begin{aligned}
& \Delta \sigma_{t}=\frac{E \alpha_{T} \Delta T}{1-v} \frac{1}{4 \pi}\left[\pi+\frac{n}{p^{2} \sqrt{(p+1)^{2}+n^{2}}}\left[\left((p+1)^{2}+n^{2}\right)(K-E)+(p-1)^{2} K\right]-\right. \\
& \left.\frac{p^{2}-1}{p^{2}}\left[K \bar{E}\left(\varphi^{\prime}, k^{\prime}\right)-\frac{K E^{\prime} \bar{K}\left(\varphi^{\prime}, k^{\prime}\right)}{K^{\prime}}+\frac{\pi}{2} \frac{\bar{K}\left(\varphi^{\prime}, k^{\prime}\right)}{K^{\prime}}\right]\right]
\end{aligned}
$$

for all points exterior points $r \leq a, r \neq 0$ and $z<0$ [M25a].

$$
\begin{aligned}
& \Delta \sigma_{t}=\frac{E \alpha_{T} \Delta T}{1-v} \frac{1}{4 \pi}\left[3 \pi-\frac{n}{p^{2} \sqrt{(p+1)^{2}+n^{2}}}\left[\left((p+1)^{2}+n^{2}\right)(K-E)+(p-1)^{2} K\right]-\right. \\
& \left.\frac{p^{2}-1}{p^{2}}\left[K \bar{E}\left(\varphi^{\prime}, k^{\prime}\right)-\frac{K E^{\prime} \bar{K}\left(\varphi^{\prime}, k^{\prime}\right)}{K^{\prime}}+\frac{\pi}{2} \frac{\bar{K}\left(\varphi^{\prime}, k^{\prime}\right)}{K^{\prime}}\right]\right]
\end{aligned}
$$

for all interior points $r \leq a, r \neq 0$ and $z \geq 0$ [M25b].

$\Delta \sigma_{t}=\Delta \sigma_{r}=\frac{1}{4} \frac{E \alpha_{T} \Delta T}{1-v}\left[1+\frac{n}{\sqrt{1+n^{2}}}\right]$

for all points along the z-axis with $r=0$ and $z<0$ [M26].

$\Delta \sigma_{t}=\Delta \sigma_{r}=-\frac{1}{4} \frac{E \alpha_{T} \Delta T}{1-v}\left[3-\frac{n}{\sqrt{1+n^{2}}}\right]$

for all points along the $z$-axis with $r=0$ and $z \geq 0$ [M26a]. 


\section{References}

1. Baisch, S., Vörös, R., Rothert, E., Stang, H., Tischner, T., and R. Schellschmidt: A numerical model for fluid injection induced seismicity at Soultz-sous-Forêts. Int. Jour. Rock Mech. Min. Sci, doi:10.1016/j.ijrmms.2009.10.001.

2. Candela, T., and P. Fokker. Thermo- Poro- Elastic Stressing And Time Dependent Earthquakes Nucleation: A Semi-Analytical Injection Model. (2017). Proc. 51st US Symposium on Rock Mechanics and Geomechanics. San Francisco. June 25-28.

3. Catalli, F., Cocco, M., Console, R., Chiaraluce, L., Modeling seismicity rate changes during the 1997 Umbria-Marche sequence (central Italy) through a rate and statedependent model. J. Geophys. Res. Solid Earth., doi:10.1029/2007JB005356.

4. Chang, K. W., and P. Segall (2016), Seismicity on Basement Faults Induced by Simultaneous Fluid Injection-Extraction, Pure Appl. Geophys., doi: 10.1007/s00024016-1319-7.

5. Chen, J., and Spiers, C.J. (2016). Rate and state frictional and healing behavior of carbonate fault gouge explained using microphysical model. Journal of Geophysical Research: Solid Earth, 121 (12), (pp. 8642-8665).

6. Dieterich, J. H. (1994), A constitutive law for rate of earthquake production and its application to earthquake clustering, J. Geophys. Res., 99, 2601- 2618, doi:10.1029/93JB02581.

7. Dieterich, J. H., and B. Kilgore (1996), Implications of fault constitutive properties for earthquake prediction, Proc. Natl. Acad. Sci., 93(9),3787-3794.

8. Fjaer, E., Holt, R.M., Horsrud, P., Raaen, A.M., Risnes, R. (2008), Petroleum related rock mechanics $2^{\text {nd }}$ edition, Elsevier.

9. Geertsma, J. (1973a). A basic theory of subsidence DUE to reservoir compaction: The homogeneous case. Verhandelingen Koninklijk Nederlandsch Geologisch Mijnbowkundig Genootschap 2S, 43-61.

10. Izadi, G., Elsworth, D. (2015) The influence of thermal-hydraulic-mechanical-and chemical effects on the evolution of permeaility, seismicity and heat production in geothermal reservoirs. Geothermics. Vol 53. pp. 385-395.

11. Jaeger, J.C., Cook, N.G., and Zimmerman, R. (2007). Fundamentals of rock mechanics. John Wiley \& Sons.

Jalali, M.R. (2013). Thermo-Hydro-Mechanical Behavior of Conductive Fractures using a Hybrid Finite Difference - Displacement Discontinuity Method. UWSpace. http://hdl.handle.net/10012/7642.

12. Marone, C. (1998), Laboratory-derived friction laws and their application to seismic 
faulting, Ann. Rev. Earth Planet. Sci., 26, 643-696.

13. McClure, M.W., Horne, R.N. Discrete Fracture Modeling of Hydraulic Stimulation in Enhanced Geothermal Systems. Proceedings, Thirty-Fifth Workshop on Geothermal Reservoir EngineeringStanford University, Stanford, California, February 1-3, 2010 SGP-TR-188.

14. Mindlin, R.D., 1936. Force at a point in the interior of a semi-infinite solid, Physics, 7(5), 195-202.

15. Mindlin, R. D. and Cheng, D. H. (1950). Thermo-Elastic Stress in the Semi-Infinite Solid," J. Applied Phys. 21, 931.

16. Mossop, A., Injection Induced Seismicity: A thermoelastic model, Ph.D. thesis, $\begin{array}{lll}\text { chapter } & 5, & 2001\end{array}$ (https://pangea.stanford.edu/cdfm/sites/default/files/pubs/mossop2001ch5.pdf).

17. Myklestad, N.O. (1942). Two problems of thermal stress in the infinite solid, Journal of Applied Mechanic, 9, 136-143.

18. Niemeijer, A.R., and Spiers, C.J. (2007). A microphysical model for strong velocity weakening in phyllosilicate-bearing fault gouges. Journal of Geophysical Research: Solid Earth, 112 (B10), (pp. B10405) (1 p.).

19. Nikkhoo, M., Walter, T. R., Lundgren, P. R., Prats-Iraola, P. (2016): Compound dislocation models (CDMs) for volcano deformation analyses. - Geophysical Journal International, 208, 2, p. 877-894.

20. Okada, Y., 1985. Surface deformation due to shear and tensile faults in a half-space, Bull. seism. Soc. Am., 75(4), 1135-1154.

21. Okada, Y., 1992. Internal deformation due to shear and tensile faults in a half-space, Bull. seism. Soc. Am., 82(2), 1018-1040.

22. Segall, P. (1992). Induced stresses due to fluid extraction from axisymmetric reservoirs. Pure and Applied Geophysics, 139(3-4), 535-560.

23. Segall, P., and S. Lu (2015), Injection-induced seismicity: Poroelastic and earthquake nucleation effects, J. Geophys. Res. Solid Earth, 120, 5082-5103, doi:10.1002/2015JB012060.

24. Sen, B. (1950). Note on the Stress Produced Nuclei of Thermoplastic Strain in a SemiInfinite Elastic Solid. Quarterly Applied Math. 8, 635.

25. Taron, J. Elsworth, D., Min, K.-B. (2009) Numerical simulation of thermal-hydrologic mechanical-chemical processes in deformable fractured porous media. Int. J. R. Mechs. Vol. 46, pp. 842-854. 
26. Theis., C.V. The relation between the lowering of the piezometric surface and the rate and duration of a well using groundwater storage. Trans. AGU, 2:519-524, 1935.

27. Zang, A., Seok Yoon, J., Stephansson, O., Heidbach, O., Fatigue hydraulic fracturing by cyclic reservoir treatment enhances permeability and reduces induced seismicity. Geophys. J. Int. doi: 10.1093/gji/ggt301. 